\title{
Gain spectroscopy of Solution Based Semiconductor Nanocrystals in Tunable Optical Microcavities
}

Robin K. Patel ${ }^{1}$, Aurélien A. P. Trichet ${ }^{1}$, David Coles ${ }^{1}$, Philip R. Dolan ${ }^{1}$, Simon Fairclough ${ }^{4}$, Marina A. Leontiadou $^{5}$, Edman Tsang ${ }^{2}$, David Binks ${ }^{5}$, Eunjoo Jang ${ }^{2}$, Hyosook Jang ${ }^{2}$, Robert A. Taylor ${ }^{3}$ and Jason M. Smith ${ }^{1}$

${ }^{1}$ Department of Materials, University of Oxford, Parks Road, Oxford OX1 3PH, UK

${ }^{2}$ Samsung Advanced Institute of Technology, Samsung Electronics Co, Suwon, Rep. Korea.

${ }^{3}$ Department of Physics, University of Oxford, Parks Road, Oxford OX1 3PU, UK

${ }^{4}$ Inorganic Chemistry Laboratory, Department of Chemistry, University of Oxford, South Parks Road, Oxford, OX1 3QR, UK

${ }^{5}$ Photon Science Institute and School of Physics and Astronomy, University of Manchester, Manchester M13 9PL, UK

ABSTRACT: We report on the lasing behaviour of solution-based colloidal quantum dots within an open microcavity. The small size and wide tunability of the cavity provide single mode lasing over a wavelength range in excess of $25 \mathrm{~nm}$. By extracting the lasing threshold and differential gain for the fundamental cavity mode over this spectral range we demonstrate gain spectroscopy of the quantum dot solution. This new approach could help in the optimisation of laser gain media and provides a way of constructing miniature laser arrays for on-chip integration.

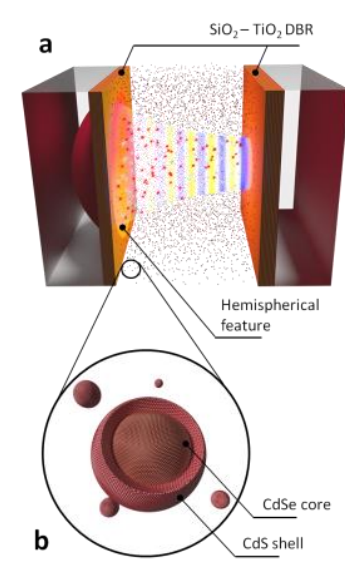

Solution processed nanocrystal quantum dots (NQDs), have since their first manufacture ${ }^{[1]}$ attracted considerable interest for use as optical gain media, with the prospect of inexpensive, low threshold laser devices that can be printed onto large area or flexible substrates, or otherwise integrated into epitaxial or waveguide structures. The quantum confinement of electrons and holes in such nanocrystals allows their emission wavelength to be tuned simply by changing their size, such that any emission wavelength in the visible and near infrared of the spectrum can be accessed with a small selection of materials. Several nanocrystal based lasers have now been reported with a variety of device designs[2]-[9].

Understanding the interplay between the various absorption and emission processes is essential in order to optimise the nanocrystal design. Spectroscopy of the gain produced can be measured using pump-probe techniques ${ }^{10]}$ and allows the investigation of exciton relaxation dynamics. However these rapid transient processes are considerably removed from lasing behaviour in the presence of optical feedback and a good understanding of the lasing process is currently lacking. 
In this paper we report a convenient method for the direct investigation of the lasing behaviour of solution-based NQDs by performing gain spectroscopy enabled via in-situ tuning of the cavity feedback wavelength. Tunable cavities and laser emission have been demonstrated by others using various methods. For example, the shape of the cavity can be tuned by external heating achieved through either modulating the pump power ${ }^{[8]}$ or by directly heating the cavity using an electrical heater ${ }^{[11]}$. Other methods have utilised $\operatorname{strain}^{[12]}$, electric-field ${ }^{[13]-[14]}$ or pressure ${ }^{[15]}$. However, these methods tend to either have too limited a tuning range for useful spectroscopy, or involve changes to the NQD environment that may themselves modify the gain characteristics, making interpretation of the observed changes in lasing performance difficult. By directly tuning the cavity length of a FabryPerot style open resonator, we achieve both a wide tuning range and fine control limited only by the resolution of the actuator. As a result we are able to observe single mode lasing over a range exceeding $25 \mathrm{~nm}$, and thereby map the full spectra for the lasing threshold and differential gain. The NQDs in solution within the cavity experience no change in temperature or stress during tuning and so the changes in lasing performance can be attributed unambiguously to the change in feedback wavelength. From a technological standpoint, our results demonstrate a new approach to widely tunable single mode lasers for chip-scale and low power applications. 

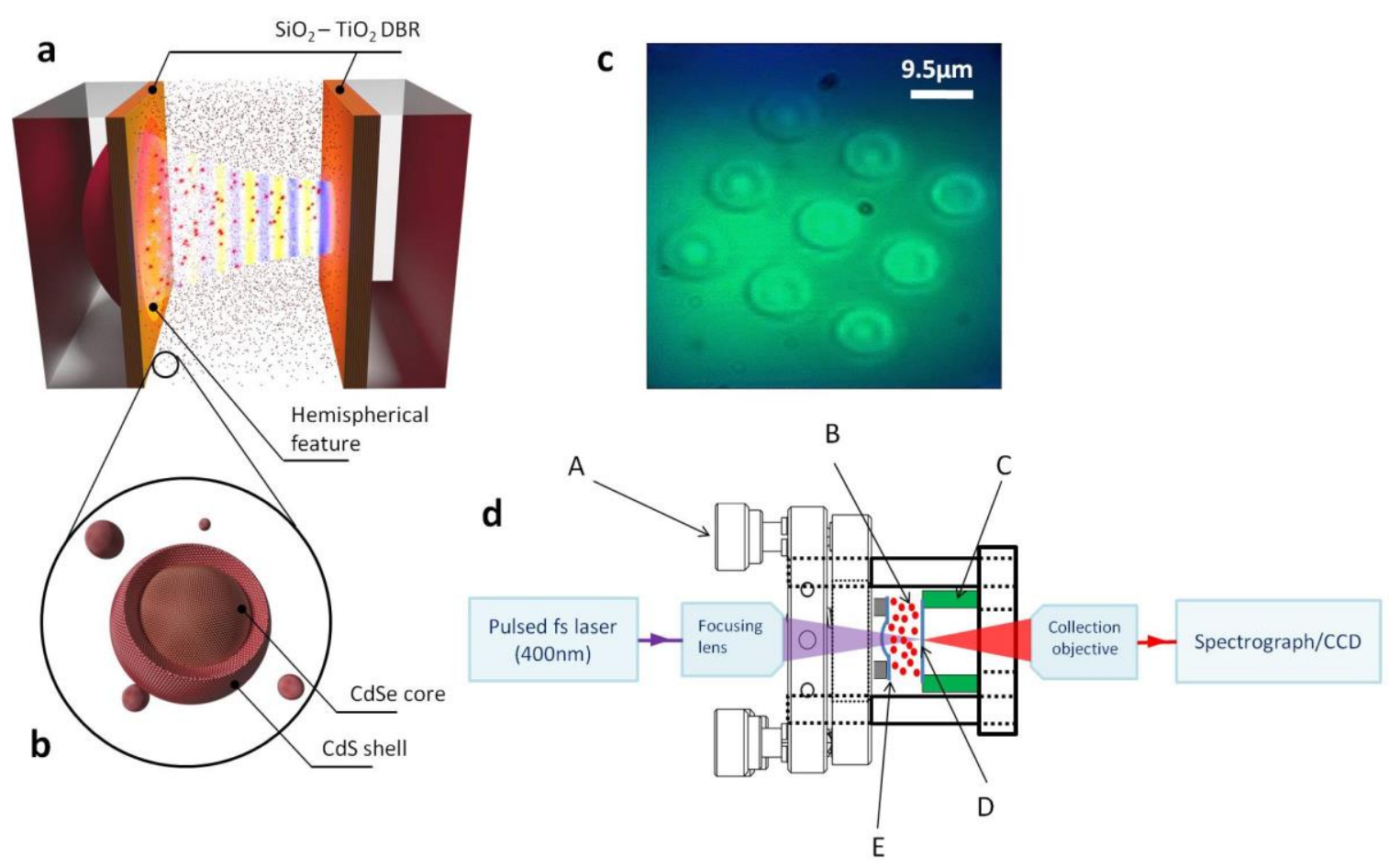

Figure 1. Cavity geometry and set-up. (a) Diagram showing the cavity which consists of a hemispherical and a flat mirror each made from 10 alternating pairs of $\lambda / 4 \quad \mathrm{SiO}_{2} / \mathrm{TiO}_{2}$ material forming the distributed bragg reflectors (DBRs). The hemispherical mirror has a radius of curvature of $25 \mu \mathrm{m}$. (b) Core-shell CdSe/CdS NQD solution inside the cavity. (c) Image showing $3 \times 3$ array of cavities (patterned mirror) of $25 \mu \mathrm{m}$ radius of curvature. (d) Schematic of the experimental set-up. $\mathrm{A}=$ Tilt control, $\mathrm{B}=$ Nanocrystal solution, $\mathrm{C}=$ Piezo ring actuator, $\mathrm{D}=$ Planar mirror, $\mathrm{E}=$ Patterned mirror. The NQDs are excited with a pulsed $400 \mathrm{~nm}$ laser with $100 \mathrm{fs}$ temporal width and a $0.4 \mathrm{NA}$ objective is used to focus the laser on the cavity. The emission from these NQDs is collected using a $0.25 \mathrm{NA}$ objective and directed into the spectrometer. The cavity length is controlled by a ring piezoelectric actuator and the tilt control allows us to tune the angle between the two mirrors during alignment.

The experimental setup is shown in figure 1. The cavity consists of a planar mirror and a spherical concave mirror facing each other as shown in figure 1a. The concave mirror templates are manufactured from a polished silica substrate using ion beam milling (FIB) as reported in previous work $^{[16]}$. Each mirror is then coated with distributed Bragg reflectors (DBRs) with a maximum reflectivity of approximately $99.87 \%$ at $640 \mathrm{~nm}$ and a $>90 \%$ reflectivity band extending from $550 \mathrm{~nm}$ to $720 \mathrm{~nm}$. Each DBR is made up of 10 pairs of $\mathrm{SiO}_{2} / \mathrm{TiO}_{2}$. The hemispherical mirrors used in this work have a nominal radius of curvature $(\mathrm{RoC})$ of $25 \mu \mathrm{m}$, providing a confined mode diameter of 1-2 $\mu \mathrm{m}$ depending on cavity length. The core-shell CdSe/CdS NC's (figure 1b) are immersed in toluene and drop-cast onto the planar mirror and remain in the form of a solution during the experiment. The solution completely filled the cavity as shown on Figure 1a. In figure 1c a $3 \times 3$ array of hemispherical cavities (patterned mirror) can be seen. Figure 1d shows the cavity control apparatus and optics. The NC's are optically excited with a frequency-doubled mode locked Ti:Sapphire laser at $400 \mathrm{~nm}$, capable of delivering pulse durations of $100 \mathrm{fs}$ and with energy in excess of $700 \mathrm{pJ}$ per pulse. The 
excitation beam is focused to a spot diameter (FWHM) of $10.5 \mu \mathrm{m}(+/-1 \mu \mathrm{m})$ diameter using a 0.4 NA microscope objective lens. The repetition rate of the excitation pulses is set to $40 \mathrm{kHz}$ to avoid photobleaching of the NC's. The hemispherical mirror is attached to a 2-axis kinematic stage which allows fine control of the angle between the two mirrors. The planar mirror is attached to a piezoelectric ring actuator used to control the cavity length. Emission from the device is collected using a $0.25 \mathrm{NA}$ objective lens and is directed into a grating spectrometer with a cooled silicon CCD camera (figure 1d).

The microcavities support stable Hermite-Gaussian modes provided the mirror separation $L$ is smaller than the radius of curvature $(\beta)$ of the concave mirror. Within this stability range, each cavity mode is fully characterized by three mode numbers. The longitudinal mode number $q$ gives the number of field anti-nodes along the cavity axis and the two transverse mode number, $m$ and $n$, correspond to excitations perpendicular to the cavity axis (vertical on figure 1a) resulting from the confinement caused by the concave mirror ${ }^{[16]-[21]}$. For a given set of mode numbers $(q, m, n)$, the mode resonance wavelength $\lambda$ is given by:

$\left[q+\frac{1}{\pi}(m+n+1) \arccos (\sqrt{1-L / \beta})\right] \frac{\lambda}{2 \mu_{\mathrm{ref}}}=L$

where $\mu_{\text {ref }}$ is the refractive index. Figure 2a shows a typical emission spectra below and above the lasing threshold at pump energies $0.85 P_{\text {th }}$ and $2.66 P_{\text {th }}$ respectively. Here $P_{\text {th }}$ is the threshold energy (in pJ per pulse) of the fundamental cavity mode TEM $\mathrm{T}_{00}$ (with $m=n=0$ ) observed here at $641 \mathrm{~nm}$. This mode is associated with $q=25$ calculated using eq. (1) with a cavity length of $5.3 \mu \mathrm{m}$ inferred from the free spectral range. The refractive index is taken to be as 2 (a 50:50 mixture of toluene and CdSe/CdS NQD solution is assumed). In addition a family of transverse modes can be seen (up to about $m+n=4)$. Note that on the higher wavelength side of the fundamental mode $(q, 0,0)$ other transverse modes associated with a lower longitudinal mode number $q-1$ can also be seen.

The integrated intensity of the fundamental mode as a function of excitation energy reveals a clear threshold behaviour as presented in figure 2b. The modes at $622 \mathrm{~nm}$ and $641 \mathrm{~nm}$ display lasing thresholds of $190 \mathrm{pJ}$ and $80 \mathrm{pJ}$ respectively, as shown in figure $2 \mathrm{~b}$. Figure $2 \mathrm{~b}$ also shows their linewidths (FWHM) as a function of excitation energy which can be seen to drop sharply at threshold due to the increase in the coherence time of the system and they quickly reach the resolution limit of the spectrometer $(\sim 0.1 \mathrm{~nm})$. This sharp reduction in the linewidth provides conclusive evidence of lasing and provides the most accurate method for determining the lasing threshold. The inset shows the spectra above threshold for these two modes tuned into position one after another using the ring actuator. 

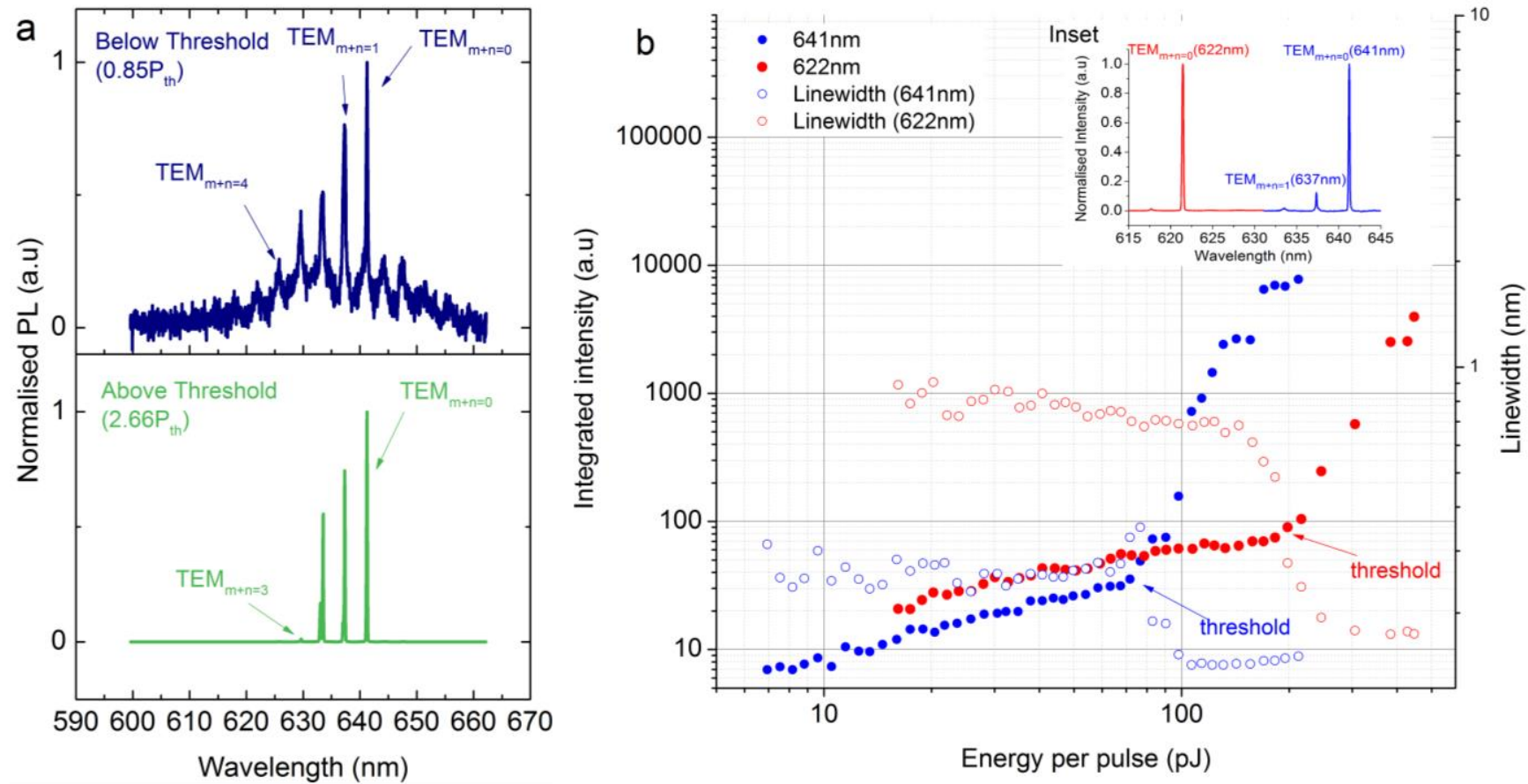

Figure 2. Nanocrystal lasing. (a) Spectra below and above threshold at pump energies of $0.85 P_{\text {th }}$ and $2.66 P_{\text {th }}(400 \mathrm{~nm}$ excitation), respectively. Multi-mode laser emission (from $\mathrm{TEM}_{\mathrm{m}+\mathrm{n}=0}$ to $\mathrm{TEM}_{\mathrm{m}+\mathrm{n}}=3$ ) can be seen above threshold. (b) Threshold behaviour from the fundamental cavity mode, $\mathrm{TEM}_{\mathrm{m}+\mathrm{n}=0}$, at two different spectral positions (622 $\mathrm{nm}$ and 641 $\mathrm{nm}$ ), along with the linewidths, showing a sharp decrease at threshold. Notice that the threshold is different for these two spectral positions ( $\sim 80 \mathrm{pJ}$ per pulse for $\operatorname{TEM}_{\mathrm{m}+\mathrm{n}}=0$ at $641 \mathrm{~nm}$ and $\sim 190 \mathrm{pJ}$ per pulse at $622 \mathrm{~nm}$ ), revealing the possibility of mapping the threshold as a function of spectrum position. The inset shows the spectra of these lasing modes at these two different spectral positions, both above threshold.

Assuming the absorption at the pump wavelength is non-saturable, we calibrate the excitation pulse energy in terms of the average number of excitons generated per NQD, $\langle n\rangle$ (see supplementary information). Then, as an additional means to characterise lasing behaviour we define a dimensionless 'differential gain parameter' $\eta$ as $\eta=\frac{d \chi}{d\langle n\rangle}$ where $\chi=\frac{P_{\mathrm{st}}}{P_{\mathrm{sp}}}$, the ratio of the stimulated emission intensity to the spontaneous emission intensity (the latter being taken to be constant at the threshold value). The parameter $\eta$ is thus measured using a linear least squares fit to the graph of $\chi$ vs $\langle n\rangle$ above the lasing threshold.

The threshold and differential gain parameters can thus be established at any wavelength within the spectral range over which lasing is observed by tuning the cavity length. Figure 3 shows the tuning of the $\mathrm{TEM}_{00}$ lasing mode from $619 \mathrm{~nm}-651 \mathrm{~nm}$, a range of $32 \mathrm{~nm}$. Note that between $619 \mathrm{~nm}-644$ $\mathrm{nm}$ we are able to select a pump power at which lasing occurs predominantly in the TEM $\mathrm{T}_{00}$ mode. Between $648 \mathrm{~nm}-651 \mathrm{~nm} \mathrm{TEM}_{\mathrm{m}+\mathrm{n}=1}$ and $\mathrm{TEM}_{\mathrm{m}+\mathrm{n}=2}$ dominate the spectrum. 


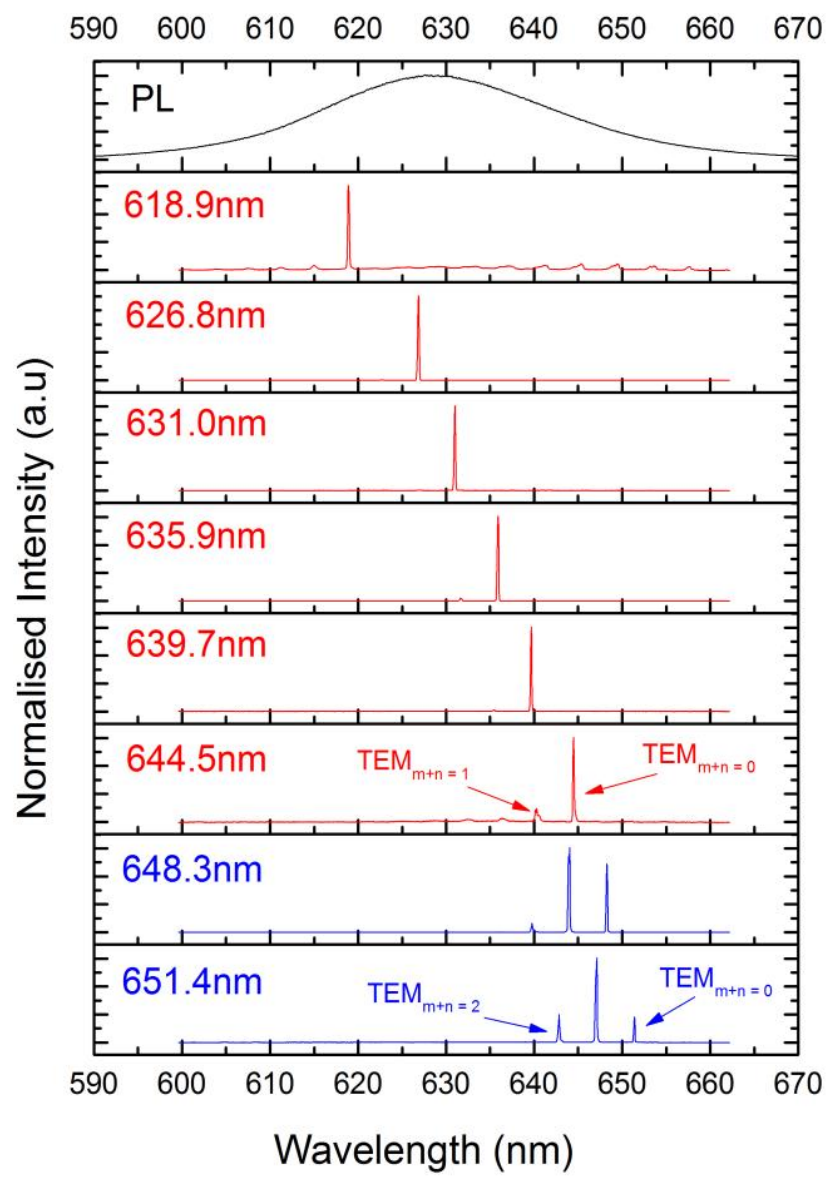

Figure 3. Tunable laser emission. The optical modes can be tuned by varying the length of the cavity. The open access design of the cavity gives us fine control of the tunability as well as a large tuning range. Single mode tunability of $25.6 \mathrm{~nm}$ is demonstrated on the fundamental mode of the cavity in the lasing regime. The photoluminescence spectrum is also plotted for comparison.

Graphs of the TEM $\mathrm{TO}_{00}$ lasing threshold, plotted in terms of the average exciton number per NQD $(\langle n\rangle)$, and differential gain parameter as functions of spectral position are shown in figure 4a on top of the normalised free space emission spectrum. The lasing threshold shows a clear minimum which is redshifted relative to the peak of the PL spectrum. This spectral position for the minimum threshold is consistent with observations in NQD's ${ }^{[5],[7],[10],[22]}$ and dye-doped laser systems[ ${ }^{23]}$ without resonant optical feedback. In these, the appearance of amplified spontaneous emission to the red of the spontaneous emission peak has been justified by the fact that the net gain is a result of the competition between stimulated emission and absorption processes in the nanocrystals, the latter always being stronger at shorter wavelengths. Note that our cavity Q-factor is governed by the reabsorption processes of the nanocrystals so that threshold curve seen in figure $4 \mathrm{~b}$ is not due to the intrinsic Q-factor of the cavities, which peaks around 640nm. The differential gain also shows a maximum at the point where the threshold is at a minimum meaning that the lasing process is more 
efficient here. The average exciton number at threshold can be calculated from the equation $\langle n\rangle=$ $J_{\mathrm{p}} \sigma / \mathrm{h} \omega_{\text {pump }}$. Here $J_{p}$ is the pump photon fluence (calculated using the excitation spot size), $\sigma$ the absorption cross-section and $\hbar \omega_{\text {pump }}$ is the pump photon energy. Taking $\sigma$ as $8 \times 10^{-19} \mathrm{~m}^{2[24]}$ and assuming efficient relaxation to the band edge $\mathrm{e}^{[\mathrm{x}]}$, the average exciton number at threshold is calculated and plotted in figure $4 \mathrm{~b}$ (black). This calculated curve agrees well with the curve for the average exciton number at threshold (blue) inferred using the saturation fit below threshold (see supplementary information).

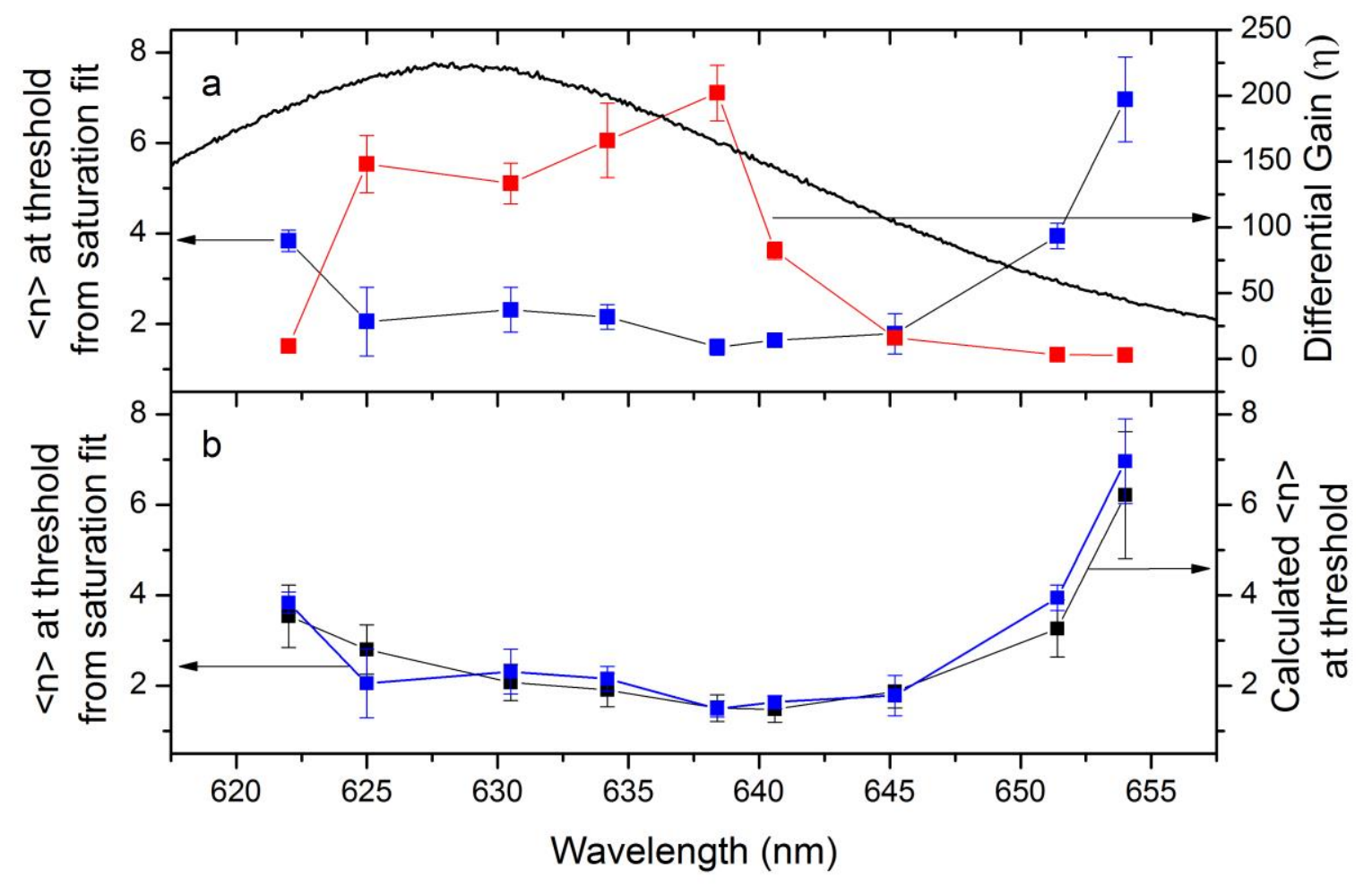

Figure 4. Lasing spectroscopy of the nanocrystal solution. (a) Threshold exciton number (solid squares) and differential gain (solid circles) as functions of the TEM 00 lasing wavelength. The nanocrystal fluorescence spectrum (solid line) is included for comparison. (b) Values of $<n>$ at threshold obtained by fitting the fluorescence saturation curve (solid triangles) and by measurement of the pump energy per pulse, assuming an absorption cross section at $\lambda_{\text {pump }}=400 \mathrm{~nm}$ of $8 \times 10^{-19} \mathrm{~m}^{2}$.

The desire for low lasing thresholds has focused much attention on the realisation of lasing in the single exciton regime ${ }^{[7],[8],[25],[26]}$. Single exciton lasing is challenging because the spin degeneracy of the lowest energy exciton state in NQDs suggests that in the absence of exciton-exciton interaction, a single exciton will not provide gain because it will offer a cross section for optical absorption equal to that of stimulated emission. In this simple picture therefore, only biexciton states are capable of producing a net gain. Some recent reports however have demonstrated that engineering the interactions between the photoexcited electrons and holes can lift the exciton/biexciton degeneracy such that lasing in the single exciton regime is observed ${ }^{[8]}$. 
Using the calibrated exciton generation axis (see supplementary infomation) we find that $<n>\sim 1.49$ $\pm(0.18)$ for the lowest measured lasing threshold suggesting that lasing is primarily generated by biexcitons in the present device. This is consistent with the known physics of the NQD structures and cavities used in the experiments: although $\mathrm{CdSe} / \mathrm{CdS}$ core-shell nanocrystals may have potential to show lasing in the single exciton regime due to their quasi-type II core-shell band alignment[8], the relatively thin shells of these NCs will result in near-degenerate exciton and biexciton transitions, and so it is likely that the stimulated emission cross section for single exciton lasing is at least an order of magnitude smaller than that for biexciton lasing, and would only be observed with a very high finesse cavity.

In conclusion, we have demonstrated in-situ tunable single mode lasing over a range in excess of 25 $\mathrm{nm}$ from solution-based semiconductor nanocrystals using our optical microcavities. Our method of tuning the lasing wavelength ensures there is no modification to the NQD environment and so the lasing performance can be attributed unambiguously to the change in feedback wavelength. The threshold of the fundamental cavity mode, $\operatorname{TEM}_{\mathrm{m}+\mathrm{n}=0}$, as a function of its spectral position was shown to have a clear minimum red-shifted with respect to the ensemble photoluminescence spectrum. This minimum was attributed to an optimal balance between stimulated emission and re-absorption of the lasing photons. We further define a differential gain parameter and measure it to be maximum where the threshold is at its minimum showing the lasing here is at its most efficient. This knowledge could help optimize cavity feedback designs for low threshold lasing and also provides a way of constructing miniature laser arrays for on-chip integration. Furthermore, the capability to manufacture an array of hemispherical cavities points towards the possibility of scaling up our miniature laser cavities for onchip integration.

\section{Supplementary Information}

\section{Saturation of spontaneous emission}

If the absorption process at the pump wavelength is non-saturable, the probability of generating $k$ excitons per NC immediately after the pump pulse follows the Poissonian distribution ${ }^{[27]}$ :

$\mathrm{P}(\mathrm{k})=\frac{\langle n\rangle^{k}}{k !} \mathrm{e}^{-\langle n\rangle}$

where $\langle n\rangle$ is the average number of excitons generated per nanocrystal, proportional to the excitation pulse energy. Since multiple exciton states in nanocrystals decay via rapid and non-radiative Auger processes $^{[28]-[31]}$, the spontaneous emission is dominated by the radiative recombination of single excitons. Consequently, the spontaneous emission intensity saturates with increasing excitation pulse energy, as the probability of generating at least one exciton per nanocrystal approaches unity. Equ. (A1) reveals that the probability of generating at least one exciton in a nanocrystal is given by

$\mathrm{P}(\geq 1)=1-e^{-\langle n\rangle}$ 
The dependence of the spontaneous emission intensity $I_{s p}$ on energy per excitation pulse $E_{e x}$ therefore takes the form

$I_{\mathrm{sp}}=P_{\mathrm{sp}}\left(1-\mathrm{e}^{-\frac{E_{\mathrm{ex}}}{E_{1}}}\right)$

where $E_{1}$ is defined as the pulse energy required per exciton and $P_{s p}$ is the spontaneous emission intensity taken to be constant at the threshold value.

By fitting eq. (A3) to the measured data we are able accurately to establish $E_{1}$. An example fit is shown in figure 1. For one of the fundamental lasing modes at $622 \mathrm{~nm}$, for which $E_{\text {sat }}=49.4 \pm 2.1 \mathrm{pJ}$ per exciton. The average number of excitons per NQD resulting from this calibration, is plotted on the top axis of figure $\mathrm{S} 1$.

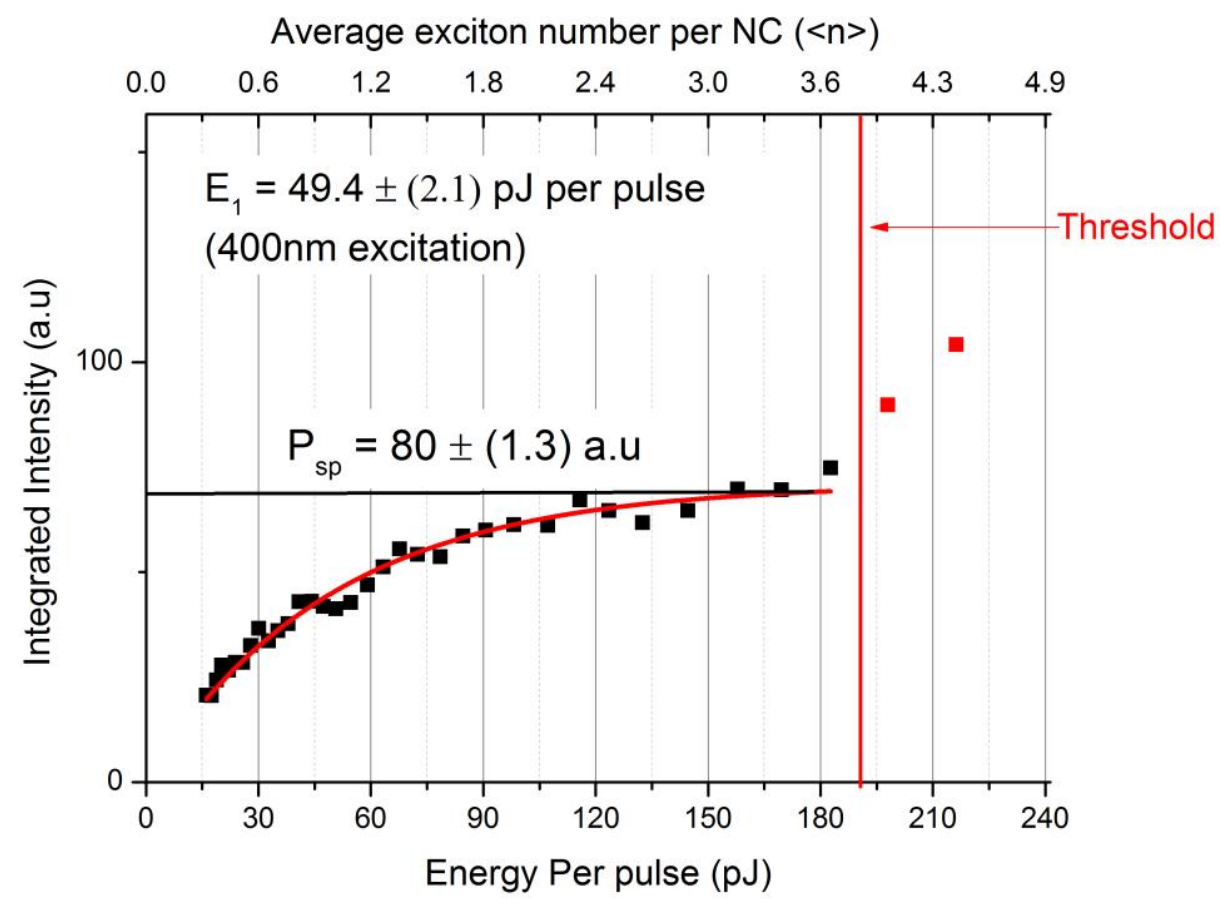

Figure S1. Fluorescence saturation curve fitting. The excitation pulse energy can be calibrated in terms of the average exciton number per NQD through equation A3. Shown here is an example fit to the power dependence curve (below threshold) for the $\mathrm{TEM}_{\mathrm{m}+\mathrm{n}=0}$ mode at $622 \mathrm{~nm}$. This enables us to extract the energy needed to generate a single exciton per NC $\left(E_{1}\right)$ and also the saturation constant $P_{s p}$, which allows us to define the differential gain parameter, $\eta$ (see main text). 
[1] C. B. Murray, D. J. Norris, and M. G. Bawendi, "Synthesis and characterization of nearly monodisperse $\mathrm{CdE}(\mathrm{E}=\mathrm{S}$, Se, Te) semiconductor nanocrystallites" J. Am. Chem. Soc. 115, pp. 8706-8715, 1993.

[2] H.-J. Eisler, V. C. Sundar, M. G. Bawendi, M. Walsh, H. I. Smith, and V. Klimov, "Colorselective semiconductor nanocrystal laser," Appl. Phys. Lett., vol. 80, no. 24, p. 4614, 2002.

[3] A. V. Malko, a. a. Mikhailovsky, M. a. Petruska, J. a. Hollingsworth, H. Htoon, M. G. Bawendi, and V. I. Klimov, "From amplified spontaneous emission to microring lasing using nanocrystal quantum dot solids," Appl. Phys. Lett., vol. 81, no. 7, p. 1303, 2002.

[4] B. Min, S. Kim, K. Okamoto, L. Yang, A. Scherer, H. Atwater, and K. Vahala, "Ultralow threshold on-chip microcavity nanocrystal quantum dot lasers," Appl. Phys. Lett., vol. 89, no. 19, p. 191124, 2006.

[5] S. Gao, C. Zhang, Y. Liu, H. Su, L. Wei, T. Huang, N. Dellas, S. Shang, S. E. Mohney, J. Wang, and J. Xu, "Lasing from colloidal InP/ZnS quantum dots.," Opt. Express, vol. 19, no. 6, pp. 5528-35, Mar. 2011.

[6] Y. Chen, B. Guilhabert, J. Herrnsdorf, Y. Zhang, A. R. MacKintosh, R. a. Pethrick, E. $\mathrm{Gu}, \mathrm{N}$. Laurand, and M. D. Dawson, "Flexible distributed-feedback colloidal quantum dot laser," Appl. Phys. Lett., vol. 99, no. 24, pp. 1-4, 2011.

[7] C. Dang, J. Lee, C. Breen, J. S. Steckel, S. Coe-Sullivan, and A. Nurmikko, "Red, green and blue lasing enabled by single-exciton gain in colloidal quantum dot films.," Nat. Nanotechnol., vol. 7, no. 5, pp. 335-9, May 2012.

[8] C. Grivas, C. Li, P. Andreakou, P. Wang, M. Ding, G. Brambilla, L. Manna, and P. Lagoudakis, "Single-mode tunable laser emission in the single-exciton regime from colloidal nanocrystals," Nat. Commun., vol. 4, no. May, pp. 1-9, Aug. 2013.

[9] J. Q. Grim, S. Christodoulou, F. Di Stasio, R. Krahne, R. Cingolani, L. Manna, and I. Moreels, "Continuous-wave biexciton lasing at room temperature using solutionprocessed quantum wells," Nat. Nanotechnol., vol. 9, no. 11, pp. 891-895, 2014.

[10] V. I. Klimov, a. a. Mikhailovsky, S. Xu, a Malko, J. a. Hollingsworth, C. a Leatherdale, H.-J. Eisler, and M. G. Bawendi, "Optical Gain and Stimulated Emission in Nanocrystal Quantum Dots," Science (80-. )., vol. 290, no. 5490, pp. 314-317, 2000.

[11] A. Chiba, H. Fujiwara, J. Hotta, S. Takeuchi, and K. Sasaki, "Resonant Frequency Control of a Microspherical Cavity by Temperature Adjustment," Jpn. J. Appl. Phys., vol. 43, no. 9A, pp. 6138-6141, Sep. 2004.

[12] W. Von Klitzing, R. Long, and V. Ilchenko, "Frequency tuning of the whispering-gallery modes of silica microspheres for cavity quantum electrodynamics and spectroscopy," Opt. ..., vol. 26, no. 3, pp. 166-168, 2001.

[13] D. Armani, B. Min, A. Martin, and K. J. Vahala, "Electrical thermo-optic tuning of ultrahigh-Q microtoroid resonators," Appl. Phys. Lett., vol. 85, no. 22, pp. 5439-5441, 2004.

[14] M. Humar, M. Ravnik, S. Pajk, and I. Muševič, "Electrically tunable liquid crystal optical microresonators," Nat. Photonics, vol. 3, no. 10, pp. 595-600, Sep. 2009. 
[15] R. Henze, T. Seifert, J. Ward, and O. Benson, "Tuning whispering gallery modes using internal aerostatic pressure.," Opt. Lett., vol. 36, no. 23, pp. 4536-8, Dec. 2011.

[16] P. R. Dolan, G. M. Hughes, F. Grazioso, B. R. Patton, and J. M. Smith, "Femtoliter tunable optical cavity arrays.," Opt. Lett., vol. 35, no. 21, pp. 3556-8, Nov. 2010.

[17] M. Trupke, E. a. Hinds, S. Eriksson, E. a. Curtis, Z. Moktadir, E. Kukharenka, and M. Kraft, "Microfabricated high-finesse optical cavity with open access and small volume," Appl. Phys. Lett., vol. 87, no. 21, p. 211106, 2005.

[18] G. Cui, J. M. Hannigan, R. Loeckenhoff, F. M. Matinaga, M. G. Raymer, S. Bhongale, M. Holland, S. Mosor, S. Chatterjee, H. M. Gibbs, and G. Khitrova, "A hemispherical, high-solid-angle optical micro-cavity for cavity-QED studies.," Opt. Express, vol. 14, no. 6, pp. 2289-99, Mar. 2006.

[19] T. Steinmetz, Y. Colombe, D. Hunger, T. W. Hänsch, a. Balocchi, R. J. Warburton, and J. Reichel, "Stable fiber-based Fabry-Pérot cavity," Appl. Phys. Lett., vol. 89, no. 11, p. 111110, 2006.

[20] A. Muller, E. B. Flagg, M. Metcalfe, J. Lawall, and G. S. Solomon, "Coupling an epitaxial quantum dot to a fiber-based external-mirror microcavity," Appl. Phys. Lett., vol. 95, no. 17, p. 173101, 2009.

[21] D. Hunger, T. Steinmetz, Y. Colombe, C. Deutsch, T. W. Hänsch, and J. Reichel, "A fiber Fabry-Perot cavity with high finesse," New J. Phys., vol. 12, no. 6, p. 065038, Jun. 2010.

[22] A. V. Malko, A. A. Mikhailovsky, M. A. Petruska, J. A. Hollingsworth, H. Htoon, M. G. Bawendi, and V. I. Klimov, "From amplified spontaneous emission to microring lasing using nanocrystal quantum dot solids," Appl. Phys. Lett., vol. 81, no. 7, pp. 1303-1305, 2002.

[23] W. Lu, B. Zhong, and D. Ma, "Amplified spontaneous emission and gain from optically pumped films of dye-doped polymers.," Appl. Opt., vol. 43, no. 26, pp. 5074-8, Sep. 2004.

[24] C. Zhang, J. Xu, T. Zhu, F. Zhang, Z. Tan, S. J. Schiff, H. Su, S. Gao, and A. Y. Wang, "Quantum efficiency of stimulated emission in colloidal semiconductor nanocrystal quantum dots," Phys. Rev. B - Condens. Matter Mater. Phys., vol. 80, no. 3, pp. 1-6, 2009.

[25] S. A. Ivanov, J. Nanda, A. Piryatinski, M. Achermann, L. P. Balet, I. V. Bezel, P. O. Anikeeva, S. Tretiak, and V. I. Klimov, "Light amplification using inverted core/shell nanocrystals: Towards lasing in the single-exciton regime," J. Phys. Chem. B, vol. 108, no. 30, pp. 10625-10630, 2004.

[26] R. Viswanatha, S. Brovelli, A. Pandey, S. a. Crooker, and V. I. Klimov, "Copper-doped inverted core/shell nanocrystals with 'permanent' optically active holes," Nano Lett., vol. 11, no. 11, pp. 4753-4758, 2011.

[27] V. Klimov, "Optical nonlinearities and ultrafast carrier dynamics in semiconductor nanocrystals," J. Phys. Chem. B, no. section 4, pp. 6112-6123, 2000.

[28] M. Rosenb, “_ II!B,” vol. 13, no. 96, 1996. 
[29] V. I. Klimov, "Quantization of Multiparticle Auger Rates in Semiconductor Quantum Dots," Science (80-. )., vol. 287, no. 5455, pp. 1011-1013, 2000.

[30] L.-W. Wang, M. Califano, A. Zunger, and A. Franceschetti, "Pseudopotential Theory of Auger Processes in CdSe Quantum Dots," Phys. Rev. Lett., vol. 91, no. 5, p. 056404, Jul. 2003.

[31] V. Klimov, J. McGuire, R. Schaller, and V. Rupasov, "Scaling of multiexciton lifetimes in semiconductor nanocrystals," Phys. Rev. B, vol. 77, no. 19, p. 195324, May 2008. 